\title{
Metabolism and skin diseases
}

\author{
Christos C. Zouboulis ${ }^{1}$. Constantine A. Stratakis ${ }^{2} \cdot$ George P. Chrousos $^{3}$. \\ Christian A. Koch ${ }^{4,5,6}$
}

Published online: 7 December 2016

(C) Springer Science+Business Media New York 2016

Metabolic processes play an essential role in the development and physiological function of human skin [1,2]. Thus, human skin cells contain hormone-specific receptors and are targets of several hormones exerting various effects [2]. From the viewpoint of modern endocrinology, however, the skin is not only regarded as a recipient of signals from distant glands. It is also an organized cell community, in which cells use molecular signals with endocrinologic/metabolic functions for (a) local intercellular communication (intracrinology) and (b) transmission of information to the other organs of the body. Indeed, hormones are activated in the skin cells and are released into the systemic circulation. Given its dimensions $\left(2 \mathrm{~m}^{2}, 8-\right.$ $10 \mathrm{~kg}$ ), the skin represents a quite large gland of the human organism [2].

Christos C. Zouboulis

christos.zouboulis@klinikum-dessau.de

1 Departments of Dermatology, Venereology, Allergology and Immunology, Dessau Medical Center, Auenweg 38, 06847 Dessau, Germany

2 Section on Endocrinology \& Genetics (SEGEN), Eunice Kennedy Shriver National Institute of Child Health and Human Development (NICHD), National Institutes of Health, Bethesda, MD, USA

3 First Department of Pediatrics, National and Kapodistrian University of Athens Medical School, “Aghia Sophia” Children's Hospital, Athens, Greece

4 Division of Endocrinology, Diabetes, Metabolism, University of Mississippi Medical Center, Jackson, MS, USA

5 G.V. (Sonny) Montgomery VA Medical Center, Jackson, MS, USA

6 Cancer Institute, University of Mississippi Medical Center, Jackson, MS, USA

\section{Metabolic pathways and skin functions}

In this issue of Reviews in Endocrine and Metabolic Disorders, several aspects of these complex skin functions are reviewed. Extra-gonadal steroidogenesis is mainly accomplished in the skin, which metabolizes various hormones from their precursors, while it also synthesizes de novo a broad palette of sex steroids and glucocorticoids from cholesterol [3]. Nikolakis et al. [3] review the major steroidogenic properties of human skin and suggest that steroidogenesis impairment is a cardinal factor for various pathological inflammatory conditions such as acne, rosacea, atopic dermatitis, and androgenic alopecia.

Growth hormone $(\mathrm{GH})$ receptors are found in almost all skin cell types, while the expression of insulin-like growth factor- 1 is restricted to melanocytes. Both GH excess states, as in the case of acromegaly in adults or gigantism in growing children, and GH deficiency are associated with various skin manifestations [4]. Kanaka-Gantenbein et al. [4] review the main dermatologic findings in GH dysregulation, namely skin thickening, coarsening of facial features, acrochordons, puffy hands and feet, oily skin and hyperhidrosis in GH excess, and thin, dry skin, and sweating disorder in GH deficiency. Moreover, special disorders associated with GH excess exhibit specific characteristics, such as café-au-lait spots in patients with neurofibromatosis and large café-au-lait skin regions with irregular margins in patients with McCune-Albright syndrome.

The high glucose serum levels in patients with diabetes mellitus also affect the skin among systemic organs. Makrantonaki et al. [5] indicate in their review that skin disorders can often predict the onset of diabetes. Cutaneous manifestations of diabetes mellitus can be classified in (a) skin diseases with a direct association with diabetes (necrobiosis lipoidica, diabetic dermopathy, diabetic bullae, yellow skin, 
eruptive xanthomas, perforating disorders, acanthosis nigricans, oral leucoplakia, lichen planus), (b) infections (bacterial, fungal), (c) cutaneous manifestations of diabetic complications (microangiopathy, macroangiopathy, neuropathy), and (d) skin reactions to diabetic treatments [5].

Stratakis [6] reports that characteristic skin lesions are among the most diagnostic manifestations of Cushing's syndrome. They include violaceous striae, facial acne, hirsutism, acanthosis nigricans, fungal infections, hyperpigmentation, and easy bruisability. Facial plethora (along with acne and other facial skin changes) is a typical sign of Cushing's syndrome, occurring due to increased perfusion. The severity of the manifestations does not correlate with the biochemical indices of the disease [6].

The skin is affected by neurohormonal triggers, especially those related to stress. Several skin cells produce stress and sex hormones implicating them in a local "hypothalamic-pituitary-adrenal axis." Theoharides et al. [7] review the function of the peptide corticotropin-releasing hormone and neurotensin, which are produced by skin cells and have synergistic action in stimulating and degranulating mast cells. These peptides are also elevated in the serum of patients with inflammatory skin diseases exacerbated by stress.

At last, Alexopoulos and Chrousos [8] review the bidirectional communication pathways between the brain and the skin, which play significant roles in integrating interactions among skin cells, nerves, and inflammatory cells. They also present the underlying mechanisms that link stress to the common dermatoses according to the latest scientific findings.

\section{Association of metabolic and skin disorders}

Although metabolic pathways have been progressively identified in influencing skin physiology and pathophysiology, their clinical relevance for common skin diseases, such as psoriasis, acne, and hidradenitis suppurativa, has not allowed the application of this new knowledge in everyday clinical practice.

Apart from the skin alterations, psoriasis was currently shown to be associated with the metabolic syndrome and to increase the risk of type 2 diabetes mellitus and cardiovascular disorders. Wolk and Sabat [9] review the research data of the last years, which demonstrated a dysregulated adipokine balance as an important link between inflammation, metabolic syndrome, and consequent disorders. Selective adipokines play a potential role in the metabolic comorbidity of psoriasis patients and can, therefore, become a future target of effective anti-psoriatic treatment [9].

Due to the central role of the sebaceous gland in acne pathogenesis, early research had focused on its lipogenic activity. Zouboulis et al. [10] review the less studied aspects of the sebaceous gland role in the skin, such as the regulation of cellular differentiation by transcription factors, the significance of specific lipid fractions, the endocrine, and, especially, the neuroendocrine role of the sebaceous gland, its contribution to the innate immunity and the detoxification of the skin, sebaceous stem cell biology, and its role in skin aging, which have only recently attracted the attention of researchers from different disciplines. These new findings on sebaceous gland physiology and metabolic activity may stimulate the development of novel therapeutic strategies targeting specific molecular pathways involved in the pathogenesis of inflammatory skin diseases [10].

The typical onset of the disease after puberty, the female predominance, the pre-menstrual flare-ups, and the improvement of hidradenitis suppurativa/acne inversa (HS) during pregnancy, suggest a contribution of metabolic/ endocrinologic factors to this chronic inflammatory, debilitating skin disorder [11]. Furthermore, the reported efficacy of anti-androgen treatment on HS indicates a possible involvement of androgens. Karagiannidis et al. [11] report that the metabolic syndrome, which increases the HS prevalence more than fourfold, points to possible interactions between metabolic alterations and HS development and describe the current views on hormonal dysregulation and its role in HS.

Gulliver et al. [12] present a holistic evidence-based approach which implements Level of Evidence and Strength of Recommendation for the treatment of HS. The diagnosis of HS should be made by a dermatologist or other healthcare professionals with expert knowledge in HS. All patients should be offered adjuvant therapy as needed (pain management, weight loss, tobacco use cessation, treatment of superinfections, and application of appropriate dressings). The treating physician should be familiar with disease severity scores, especially Hurley staging, physician global assessment, and others. The routine use of patient-reported outcomes, including Dermatology Life Quality Index, itch, and pain assessment (Visual Analogue Scale) is strongly recommended. The need for surgical intervention should be assessed in all patients depending upon type and extent of scarring, and an evidencebased surgical approach should be implemented. Evidencebased medical treatment of mild disease consists of topical clindamycin $1 \%$ solution/gel $2 \times /$ day for 12 weeks or tetracycline 500 p.o. $2 \times /$ day for 4 months for more widespread disease. If the patient fails to exhibit a response to treatment or for patients with moderate-to-severe disease, clindamycin 300 p.o. $2 \times /$ day with rifampicin 600 p.o. $1 \times /$ day for 10 weeks should be considered. If the patient is not improved, adalimumab $160 \mathrm{mg}$ at week $0,80 \mathrm{mg}$ at week 2 , then from week $440 \mathrm{mg}$ s.c. weekly should be administered. If improvement occurs, then therapy should be maintained as long as HS lesions are present. If the patient fails to exhibit a response, then a consideration of second or third line therapy is required [12].

Abdel Naser and Zouboulis [13] review the association of male fertility and skin diseases. Several genodermatoses 
affect the skin and the male reproductive system, commonly in the form of cryptorchidism and hypogonadism. The most relevant syndromes are associated with dyschromias, such as de Sanctis-Cacchione, congenital poikiloderma, LEOPARD, and $\mathrm{H}$ syndrome, others with ichthyosis, such as Rud, and trichothiodystrophy or a group of unrelated genodermatoses, such as ablepharon macrostomia, Coffin-Siris, Gorlin-Goltz, and Werner. Acquired skin diseases may also affect male fertility, usually in the form of orchitis or epididymal obstruction or androgen antagonists. These include infections (leprosy and HIV), autoimmune (erythema nodosum leprosum), granulomatous (sarcoidosis, Langerhans cell histiocytosis), nutritional deficiency (zinc), and malignancy. In addition, several skin disease treatments affect male fertility, such as methotrexate, irradiation, and anti-androgens [13].

Stratakis [14] reviews the Carney complex, a familial lentiginosis syndrome, which covers a wide phenotypic spectrum ranging from a benign-inherited predisposition to develop cutaneous spots not associated with systemic disease to associations with several syndromes. Carney complex is caused by PRKAR1A mutations and perturbations of the cyclic AMP-dependent protein kinase (PKA) signaling pathway. In addition to the cutaneous findings, primary-pigmented nodular adrenocortical disease, growth-hormone secreting pituitary adenoma or pituitary somatotropic hyperplasia leading to acromegaly, thyroid and gonadal tumors, including a predisposition to thyroid cancer, myxomas of the heart, breast and other sites, psamommatous melanotic schwannomas, and a predisposition to a variety of cancers have been described [14].

Hannah-Shmouni et al. [15] review cutaneous flushing as a common complaint in endocrine disorders. Episodic flushing is mediated by the release of endogenous vasoactive mediators or medications, while persistent flushing results in a fixed facial erythema with telangiectasia and a cyanotic tinge owing to the large cutaneous blood vessels that contain slow-flowing deoxygenated blood. The differential diagnosis of cutaneous flushing in neuroendocrine disorders encompasses a broad spectrum of benign and malignant entities, including carcinoid syndrome, pheochromocytoma, Cushing's syndrome, medullary thyroid cancer, and pancreatic neuroendocrine tumors [15].

\section{Metabolic diseases and skin tumors}

Not only mutations but also metabolic dysregulations and chronic inflammation can lead to the development of skin and other tumors. In a further review, Stratakis [16] reports on Peutz-Jeghers syndrome, Carney complex, Cowden disease, neurofibromatosis type-1 and tuberous sclerosis as multiple endocrine neoplasias
(MEN), since they predispose to a variety of endocrine tumors and characteristic skin manifestations [16].

Karimi et al. [17] report on the dramatic increase in incidence of both obesity and many cancers, including skin cancer, and review the literature linking obesity to melanoma and non-melanoma skin cancer. The molecular mechanisms underlying the association between obesity and skin cancer are not clearly understood but emerging evidence points to changes in the tumor microenvironment including aberrant cell signaling and genomic instability in the chronic inflammatory state many obese individuals experience [17].

Reichrath et al. [18] report on the importance of skin for vitamin $\mathrm{D}$ synthesis and the consequences of vitamin D deficiency. Approximately $90 \%$ of all needed vitamin $\mathrm{D}$ has to be photosynthesized in the skin through the action of ultraviolet-B irradiation. The skin represents a key organ of the human body's vitamin D endocrine system, being both the site of vitamin D synthesis and a target tissue for biologically active vitamin D metabolites. Numerous studies, including cDNA microarray analyses of mRNAs, indicate that as many as 500-1000 genes may be regulated by vitamin D receptor ligands that control various cellular functions including growth, differentiation, and apoptosis. The induction of terminal differentiation and inhibition of the proliferation of human keratinocytes has resulted in the use of vitamin D analogs for the treatment of psoriasis. Moreover, the regulation of the vitamin $\mathrm{D}$ endocrine system is relevant to the management of inflammatory and malignant skin diseases [18].

Wissner Greene et al. [19] review systemic mastocytosis, which is characterized by accumulation of clonal, neoplastic proliferations of abnormal mast cells in one or more organ systems including the skin. The presence of D816V (KIT) mutation and elevated serum tryptase levels are minor criteria for diagnosis. The manifestations of systemic mastocytosis depend on the degree of mast cell proliferation, activation, and degranulation with variable prognosis and presentation, from indolent to "smoldering" to life-threatening disease. Particularly, bone manifestations can include osteopenia with or without lytic lesions, osteoporosis with or without atraumatic fracture, osteosclerosis with increased bone density, and isolated lytic lesions leading to increased risk of bone fracture. Treatment of systemic mastocytosis is generally palliative [19].

\section{Skin health and aging: nutritional and environmental metabolic factors}

Aging is a complex process, characterized by a progressive loss of function in multiple tissues, which leads to an increased probability of death. Kanaki et al. [20] review the 
environmental aging promoters and their individual roles in aging and point out the need of biomarker development for the elucidation of pathogenesis and the introduction of individual preventive measures. Because of the complexity of skin aging, the discovery of biomarkers is still a major research challenge; however, several biomarkers already have been identified and studied [20].

Pappas et al. [21] summarize the long association between nutrition and skin health, including all possible aspects from beauty to the aging process. Multiple pathways within skin biology are associated with the onset and clinical course of various common skin diseases, such as acne, atopic dermatitis, aging, but also with photo-protection. These conditions are critically affected by nutritional patterns and dietary interventions; well documented studies have demonstrated beneficial effects of essential nutrients on impaired skin structural and functional integrity, while they have restored skin appearance and health [21].

Last, Ju and Zouboulis [22] review the endocrinedisrupting chemicals and their ability to disrupt the production and actions of hormones through direct or indirect interaction with hormone receptors and their effects on the skin as a result of individual occupational, dietary, or environmental exposure. Endocrine-disrupting chemicals perturb the endocrine system by activating the aryl hydrocarbon receptor signal pathway and can be immunotoxic and/or carcinogenic to human skin, inducing inflammatory and allergic skin diseases, chloracne, disorders of skin pigmentation, skin cancer, and skin aging.

We had a great time putting the different parts on Metabolism and skin diseases together, we hope that you will find this thematic issue interesting and wish you a pleasant and informative reading.

Acknowledgments The contribution of Dr. C.A. Stratakis to this work was supported by the Intramural Research Program, NICHD, NIH, USA.

\section{Compliance with ethical standards}

Conflict of interest The authors declare that they have no conflict of interest.

\section{References}

1. Zouboulis CC, Bornstein SR. Endocrinology of the skin - a promising joint adventure. Horm Metab Res. 2007;39:69-70.

2. Zouboulis CC. The skin as an endocrine organ. Dermatoendocrinol. 2009;1:250-2.
3. Nikolakis G, Stratakis CA, Kanaki T, Slominski A, Zouboulis CC. Skin steroidogenesis in health and disease. Rev Endocr Metab Disord. 2016. doi:10.1007/s11154-016-9390-z.

4. Kanaka-Gantenbein C, Kogia C, Abdel-Naser MB, Chrousos GP. Skin manifestations of growth hormone-induced diseases. Rev Endocr Metab Disord. 2016. doi:10.1007 /s11154-016-9378-8.

5. Makrantonaki E, Jiang D, Hossini AM, et al. Diabetes mellitus and the skin. Rev Endocr Metab Disord. 2016. doi:10.1007/s11154016-9373-0.

6. Stratakis CA. Skin manifestations of Cushing's syndrome. Rev Endocr Metab Disord. 2016. doi:10.1007/s11154-016-9399-3.

7. Theoharides TC, Stewart JM, Taracanova A, Conti P, Zouboulis CC. Neuroendocrinology of the skin. Rev Endocr Metab Disord. 2016. doi:10.1007/s11154-016-9369-9.

8. Alexopoulos A, Chrousos GP. Stress-related skin disorders. Rev Endocr Metab Disord. 2016. doi:10.1007/s11154-016-9367-y.

9. Wolk K, Sabat R. Adipokines in psoriasis: an important link between skin inflammation and metabolic alterations. Rev Endocr Metab Disord. 2016. doi:10.1007/s11154-016-9381-0.

10. Zouboulis CC, Picardo M, Ju Q, et al. Beyond acne: current aspects of sebaceous gland biology and function. Rev Endocr Metab Disord. 2016. doi:10.1007/s11154-016-9389-5.

11. Karagiannidis I, Nikolakis G, Sabat R, Zouboulis CC. Hidradenitis suppurativa/acne inversa: an endocrine skin disorder? Rev Endocr Metab Disord. 2016. doi:10.1007/s11154-016-9366-z.

12. Gulliver W, Zouboulis CC, Prens E, Jemec GB, Tzellos T. Evidence-based approach to the treatment of hidradenitis suppurativa/acne inversa, based on the European guidelines for hidradenitis suppurativa. Rev Endocr Metab Disord. 2016. doi:10.1007/s11154-016-9328-5.

13. Abdel-Naser MB, Zouboulis CC. Male fertility and skin diseases. Rev Endocr Metab Disord. 2016. doi:10.1007/s11154-016-9368-x.

14. Stratakis CA. Carney complex: a familial lentiginosis predisposing to a variety of tumors. Rev Endocr Metab Disord. 2016. doi:10.1007/s11154-016-9400-1.

15. Hannah-Shmouni F, Stratakis CA, Koch CA. Flushing in (neuro)endocrinology. Rev Endocr Metab Disord. 2016. doi:10.1007/s11154-016-9394-8.

16. Stratakis CA. Hereditary syndromes predisposing to endocrine tumors and their skin manifestations. Rev Endocr Metab Disord. 2016. doi:10.1007/s11154-016-9401-0.

17. Karimi K, Lindgren TH, Koch CA, Brodell RT. Obesity as a risk factor for malignant melanoma and non-melanoma skin cancer. Rev Endocr Metab Disord. 2016. doi:10.1007/s11154-016-9393-9.

18. Reichrath J, Zouboulis CC, Vogt T, Holick MF. Targeting the vitamin D endocrine system (VDES) for the management of inflammatory and malignant skin diseases: an historical view and outlook. Rev Endocr Metab Disord. 2016. doi:10.1007/s11154-016-9353-4.

19. Wissner Greene L, Asadipooya A, Freitas Corradi P, Akin C. Endocrine manifestations of systemic mastocytosis in bone. Rev Endocr Metab Disord. 2016. doi:10.1007/s11154-016-9362-3.

20. Kanaki T, Makrantonaki E, Zouboulis CC. Biomarkers of skin aging. Rev Endocr Metab Disord. 2016. doi:10.1007/s11154-0169392-x.

21. Pappas A, Liakou A, Zouboulis CC. Nutrition and skin. Rev Endocr Metab Disord. 2016. doi:10.1007/s11154-016-9374-Z.

22. Ju Q, Zouboulis CC. Endocrine-disrupting chemicals and skin manifestations. Rev Endocr Metab Disord. 2016. doi:10.1007/s11154016-9371-2. 


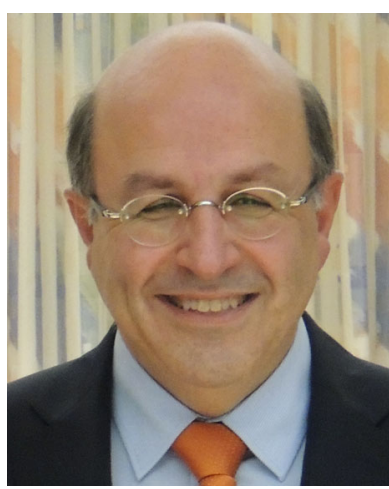

Prof. Dr. med. Christos Zouboulis has studied medicine and political sciences at the National and Kapodistrian University of Athens, Greece. He performed his pre-doctoral work on cell biology at the Department of Dermatology, Benjamin Franklin Medical Center, Freie Universitaet Berlin, Germany where he was awarded the degree Dr. med. after completing a doctoral thesis in 1988. He further continued his postgraduate medical education and completed residencies in Dermatology and Venereology as well as in Allergology at the same department. Subsequently, Dr. Zouboulis joined the Department of Dermatology, University of Michigan Medical Center, Ann Arbor, MI, USA for a research fellowship on molecular biology and the Department of Dermatology, Cleveland Clinic Foundation, Cleveland, OH, USA as well as later the Department of Dermatology, Massachusetts General Hospital, Harvard University, Boston, MA, USA for clinical research, before returning as a faculty member at the Department of Dermatology, Freie Universitaet Berlin, where he accomplished his habilitation thesis (Priv.Doz.) in 1995. In 2000, he was named Professor of Dermatology and Venereology of the Freie Universitaet Berlin, where he served as Vice Chair of the Department of Dermatology from 2000 to 2005. In 2005, he was elected Director of the Departments of Dermatology, Venereology, Allergology, and Immunology at the Dessau Medical Center, Dessau, Germany and in 2016 Founding Professor of Dermatology and Venereology at the Theodor Fontane Medical University of Brandenburg, Germany. Prof. Zouboulis is currently the Editor of the European Academy of Dermatology and Venereology (EADV) News, Co-Editor of DermatoEndocrinology and Rejuvenation Research and serves on the Editorial Boards of several leading international scientific journals. He has received numerous awards for his scientific work, including the Oskar Gans Prize of the German Dermatological Society, the EADV Research Fellowship, the Felix Wankel Animal Protection Research Prize, the Paul Gerson Unna Prize on immunological research, and the Springer Prize for Dermatology. His Departments have been decorated with the title "Germany land of ideas-2009 place of excellence" for the work on skin stem cell biology. Prof. Zouboulis was elected Prof. h.c. of the University of Franche-Comté, Besançon, France, Dr. h.c. of the National and Kapodistrian University of Athens, and Visiting Professor of the Jiao Tong University of Shanghai, China. He is an honorary member of the French, the Hungarian, and the Lithuanian Dermatological Societies. He currently serves as President of the European Hidradenitis Foundation (EHSF) e.V., as Honorary President of the European Society of Preventive, Regenerative and Anti-Aging Medicine (ESAAM) after a 10year active presidency and as Board member of the EADV and the International Society on Behçet's Disease. Prof. Zouboulis has performed fundamental research - among other fields - on Dermato-Endocrinology and sebaceous glands, acne, hidradenitis suppurativa/acne inversa, skin stem cells, molecular aging, dermato-pharmacology, cryosurgery, and rare diseases incl. Adamantiades-Behçet's disease. He is the author of more than 700 publications (cumulative impact factor 1432; h index 77), has given over 500 invited lectures, has organized numerous conferences, and is the owner on six patents, including one for the SZ95 sebaceous gland cell line, an in vitro model for acne, aging, and lipid research. He has first described the flashing pulsating angioma and the trias of keratosis pilaris, ulerythema ophryogenes, and 18p monosomy. The latter has been confirmed by several other authors and has been proposed as "Zouboulis syndrome."

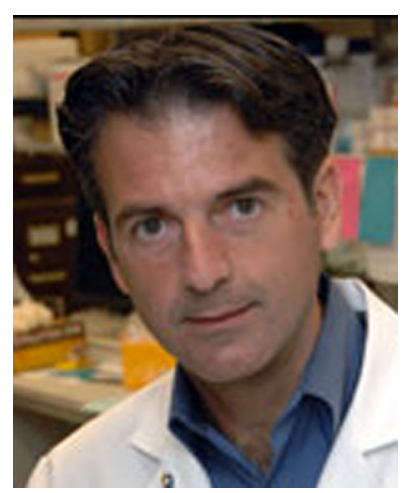

Constantine A. Stratakis received his M.D. and Doctor of Medical Sciences degrees from the National and Kapodistrian University of Athens; he did pre-doctoral work at the Unit of Endocrinology, Department of Experimental Pharmacology of the same University and briefly at the Hospital Cochin, Paris, France, before joining the Developmental Endocrinology Branch of the National Institute of Child Health \& Human Development (NICHD) at the National Institutes of Health (NIH), Bethesda, MD (1989-1990). He continued his postgraduate medical education at Georgetown University Medical School, Washington, D.C., where he finished a residency in Pediatrics and two fellowships, in Pediatric Endocrinology and Medical Genetics (in a joined program with the then newly founded National Center for Genomic Research, now NHGRI). After serving as faculty at Georgetown University Department of Pediatrics and the Fairfax Children's Hospital, he joined the Developmental Endocrinology Branch, NICHD, NIH as a Unit Chief in 1996; he continued there as a Senior Investigator and Chief of the Section on Genetics \& Endocrinology. Between 2002 and 2014, he served as the Director of the NIH Inter-Institute Pediatric Endocrinology Training Fellowship Program. From 2003 to 2011 he served first as Branch Chief and then Head of the Program on Developmental Endocrinology \& Genetics (PDEGEN), the largest on-site Department of the Intramural Clinical Research program of NICHD. Dr. Stratakis is currently serving in the Editorial Boards of several leading journals. He served (2010-15) as the Deputy Editor of the Journal of Clinical Endocrinology \& Metabolism, the leading journal in Endocrinology. Dr. Stratakis received the 1999 Pharmacia-Endocrine Society Award for Excellence in Published Clinical Research; he is the recipient of three NIH Merit Awards and other honors. He has been named Visiting Professor in several academic centers around the world and most recently received a Doctoris Honoris Causa by the Liege University, Belgium. Dr. Stratakis was awarded the 2009 Ernst Oppenheimer Award of the Endocrine Society. From 2009 to 2011, Dr. Stratakis served as the acting Scientific Director, NICHD. He is now the Scientific Director of NICHD (since August 2011), one of the largest Institutes of NIH. Dr. Stratakis is a physician scientist who has supported widely many new training programs and the professional development of junior physician scientists. Along with others, he worked on the opening of the NIH Clinical Research Center to extramural scientists starting 2013-2014; for this effort, he was the co-recipient of a $2013 \mathrm{NIH}$ Director's Award. He received the 2015 NICHD's Mentor Award and was just elected as the 2018 President of the Society for Pediatric Research (SPR). Dr. Stratakis is the author of more than 500 publications in the fields of multiple endocrine neoplasias and other genetic and endocrine syndromes, their molecular elucidation and animal models, treatment, and management. Importantly for the field of Dermatology, Dr. Stratakis identified the genes for Carney complex (PRKAR1A, PRKACB) and participated in research involving a number of other conditions involving the skin and predisposition to tumors, from MEN1, Peutz-Jeghers syndrome, NF-1, to the recent discovery of SASH1 in lentiginosis. Thus, Dr. Stratakis has contributed greatly to our understanding of predisposition to tumors, as it relates to genetic skin conditions. 


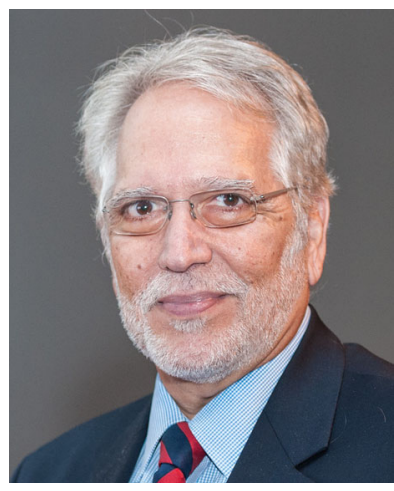

Prof. George P. Chrousos is Professor and Chairman of the First Department of Pediatrics at the National and Kapodistrian University of Athens Medical School and UNESCO Chair on Adolescent Health Care. Formerly, he was Senior Investigator, Director of the Pediatric Endocrinology Section and Training Program, and Chief of the Pediatric and Reproductive Endocrinology Branch of the National Institute of Child Health and Human Development (NICHD), National Institutes of Health. Furthermore, Dr. Chrousos has been Professor of Pediatrics, Physiology and Biophysics at Georgetown University Medical School, and John Kluge Chair on Society and Technology, Library of Congress, Washington DC, USA. In his long tenure as a researcher, Dr. Chrousos has focused his research on the hypothalamicpituitary-adrenal (HPA) axis and has extensively studied the neuroendocrine alterations associated with mood disorders, sleep, pain perception, and immune function. His clinical and laboratory program is recognized worldwide for its extensive and highly original work on the glucocorticoid signaling system, diseases of the HPA axis, such as Cushing syndrome, Addison disease, and congenital adrenal hyperplasia, and the physiologic and molecular mechanisms of stress. Early in his career, Dr. Chrousos described in the Journal of Clinical Investigation the Glucocorticoid Resistance Syndrome, a rare but unique and interesting genetic disease of the glucocorticoid receptor that causes hypertension and hyperandrogenism in children and adults.
Subsequently, Dr. Chrousos contributed the majority of the international original publications on this syndrome, which has served as a highly informative model of the study of the physiologic functions of these key steroid hormones that regulate the homeostasis of the organism and are critical for its survival. Dr. Chrousos has contributed immensely to the biomedical literature and his work has provided new insights into a spectrum of clinical conditions and disorders that transcend the limits of classical Endocrinology, such as pregnancy, human development, surgical stress, sleep, and septic shock, as well as chronic complex disorders such as depression, eating disorders, and autoimmune-inflammatory diseases. He has an outstanding record of over 1000 scientific papers and has edited 29 books, including two popular electronic volumes and two encyclopedias, and his work has been cited in about 114,000 scientific articles (h index 166), an irrefutable testimony to the importance and influence of his research on international biomedical science and clinical practice. He is one of the most cited physician scientists in the world (Institute of Scientific Information, ISI highly cited) both in the fields of Clinical Medicine and Biology and Biochemistry. In the ISI highly cited are the 250 best cited scientists and physicians by field. Clinical Medicine includes all subspecialties of Internal Medicine, Surgery, and Laboratory Medicine. Dr. Chrousos has received numerous national and international awards and has given many lectures in the USA, Europe, Latin America, and Japan. In 2014, he received the highest honor of the US Endocrine Society, the Fred Conrad Koch Award. Dr. Chrousos was inducted as a Master of both the American College of Endocrinology and the American College of Physicians and a Fellow of the Royal College of Physicians, London, UK. He is an elected member of the American Society for Clinical Research, the Association of American Physicians, the Institute of Medicine of the US National Academy of Sciences (now US National Academy of Medicine) Washington DC, USA, and the Academia Europaea, London, UK. 